Original Research Paper

\title{
Improvement of the Intelligent Tutor by Identifying the Face of the E-Learner's
}

\section{El-Kaber Hachem ${ }^{1 *}$, Moulay Hachem Harouni Alaoui ${ }^{2}$}

${ }^{1}$ Department of Physics, Faculty of Sciences, Moulay Ismail University, Meknes, Morocco

${ }^{2}$ Research Team EDP and Scientific Computing, Mathematics and Computer Department, Faculty of Science, Moulay Ismail University, Meknes, Morocco.

Article History

Received:

16.09.2019

Revised:

17.10.2019

Accepted:

27.10.2019

*Corresponding Author:

El-Kaber HACHEM

Email:

hachem.elkaber@gmail.com

This is an open access article, licensed under: $\mathrm{CC}-\mathrm{BY}-\mathrm{SA}$
Abstract: As part of our project which aims at the realization of a system named ASTEMOI. In this article, we display a new and productive facial image representation based on the Local Sensitive Hash (LSH). This technique makes it possible to recognize the learners who follow their training in our learning platform. Once recognized, the student must be oriented towards an appropriate profile that takes into account his strengths and weaknesses. We also use a light processing module on the client device with a compact code so that we don't need a lot of bandwidth, a lot of network transmission capacity to send the feature over the network, and to be able to index many pictures in a huge database in the cloud.

Keywords: Cloud, J48, Facial Image, Learning Platform, LSH. 


\section{Introduction}

As part of our project which aims at the realization of a system named ASTEMOI (Agent, composed of three agents, agent style, agent tutor, emotional agent, agent style.) We can determine the emotional status of e-learners by analysing the parameters. In the article [1,2], but in this article, we display a new and productive facial image representation based on the Local Sensitive Hash (LSH).

Automatic face analysis has become a very active research topic especially in the field of computer vision research. This analysis contains, for example, face detection, facial recognition and facial features. An essential challenge in facial analysis is finding competent descriptors for facial appearance [3].

Different holistic methods have been widely studied, such as principal component analysis (PCA) [4], linear discriminate analysis (LDA) [5], and 2D ACP [6]. Also local descriptors have received increased attention due to their strength in the face of challenges such as "pose" and "lighting changes" when taking the picture.

This article proposes an approach of the identification of students based on clouds, with Hashing Local-Sensitive. In addition, next-generation cellular networks, with their high rate of diffusion and energy expertise, provide an innovative methodology for wireless multimedia communications that joins digital technologies and wireless communications to ensure quality of service.

Even though it is very instinctive to humans, automatic learner identification is still extremely challenging due to [7]:

1. The need and ambiguity of accessible annotations;

2. Other factors, such as light, look, etc., influence the way that a face is shown in a frame;

3. Lack of data quality (low resolution, occlusion, non-rigid warp) can modify the results of face recognition and tracking.

In this paper, we present a professional approach of cloud-based student identification approach with Local-Sensitive Hashing (LSH) [8]. The LSH method is used to present "multi batch features" after detecting and tracking each face. And to know exactly the facial tracks we use "the Multi-Task Joint Sparse Representation and Classification (MTJSRC)" [9].

In order to determine the students who could have problems in their academic career, the learner must answer a questionnaire at the time of registration to collect certain information about his / her academic background, the main characteristics of his / her personality, his / her learning skills, etc.

The analysis of the mass of information gathered from the former students of the Faculty of Charia of Fez forms the basis of a predictive system which makes it possible to determine the students likely to have problems during the formation.

\section{Classification, Decision Trees J48}

\subsection{Classification}

Classification is a procedure for grouping the information gathered in specific classes as indicated by specific criteria or likeness. It requires the determination of a component that is all around portrayed to a specific class. Additionally, the classification leads to supervised learning when models are given with known class labels whereas unsupervised learning, labels are not known. Each model in the informational index is represented by an arrangement of qualities that can be characterized or continued [10]. So, classification is the way to build the model of the entire work. The remaining model is used to predict the class label of the test model.

\subsection{Decision Trees $\mathbf{J 4 8}$}

The J48 classifier is an extension of C4.5. It generates a binary tree. The decision tree is most useful in problem classification. With this technique, a tree is constructed for modelling the classification process in the decision tree, the internal nodes of the tree designate a test on an attribute, the branches represent the result of the test, the leaf node contains a label and the highest node is the root node [11] [12].

The J48 Algorithm: 


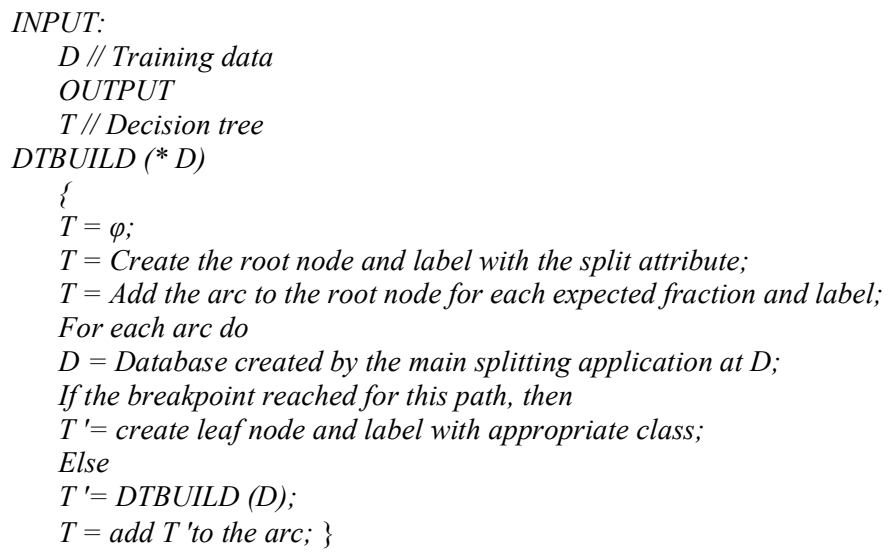

\section{Transfer of Information in Real Time}

Information continues to grow at an astounding pace. Real-time transfer of large amounts of data between source applications and data warehouses is not an easy task and will become increasingly difficult and it is essential to design flexible and optimized methods that take into account the massive growth of structured and unstructured data.

\subsection{Compact Hashing}

A solution developed for the first time by Indyk and Motwani in 1998 [13], which attracted much attention and interest from researchers, it pursues a technology called compact hashing. One of his examples is the locality-sensitive-hashing.

Locality-Sensitive Hashing is a way to not only maintain the similarity between elements, but also to reduce the dimensions of the large feature, since it hatches input elements so that similar elements map to the same Buckets with high probability.

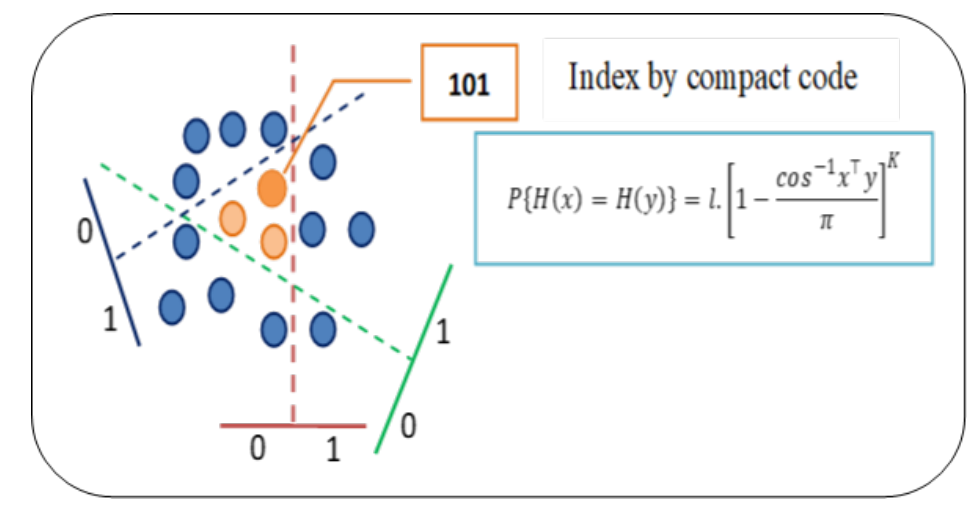

Figure 1. The Compact Hyperplanes Hashing That Allow Similar Elements to Map Into The Same Bucket

In Figure 1, we see some characteristic vectors; Blue dots. And we can consider them as a SIFT vector (Scale-invariant feature transform) [14]. So this is found in the very dimensional feature space of which we have billions of dots in high dimensional space. It can be hundreds of dimensions or even thousands of dimensions, even if we only show them on a two-dimensional screen. So the goal is to give a query point, there could be a patch from a query image user provided. 
So how can we find points of correspondence in these huge points of the database?

We consider,
$h$ : a hash function.
$x$ : the characteristic vector.
$w$ : hyperplane or protective function.

The operation of the locality-sensitive hashing is done as follows:

We project $\mathrm{x}$ in the direction of the projection function $\mathrm{w}$, and the projection value will be used to decide whether it is positive or negative by taking the threshold, which is represented by a bias value b.

For multiple hyperplanes, we can repeat this process (in this example three). Thus, each hyperplane has a normal vector, and then we assign 1 or 0 . We get a region of 101 . This compact hash code can represent a region instead of a very high dimension of functionality.

When the points are very close to each other in the original function space, their hash bits would have a very high probability of being identical. This is defined as the probability of collision, which is proportional to the original similarity.

So, if two points are very close to one another in the high- dimensional space originally, then after hashing, their binary bits are identical, and the probability is proportional to their similarity [15].

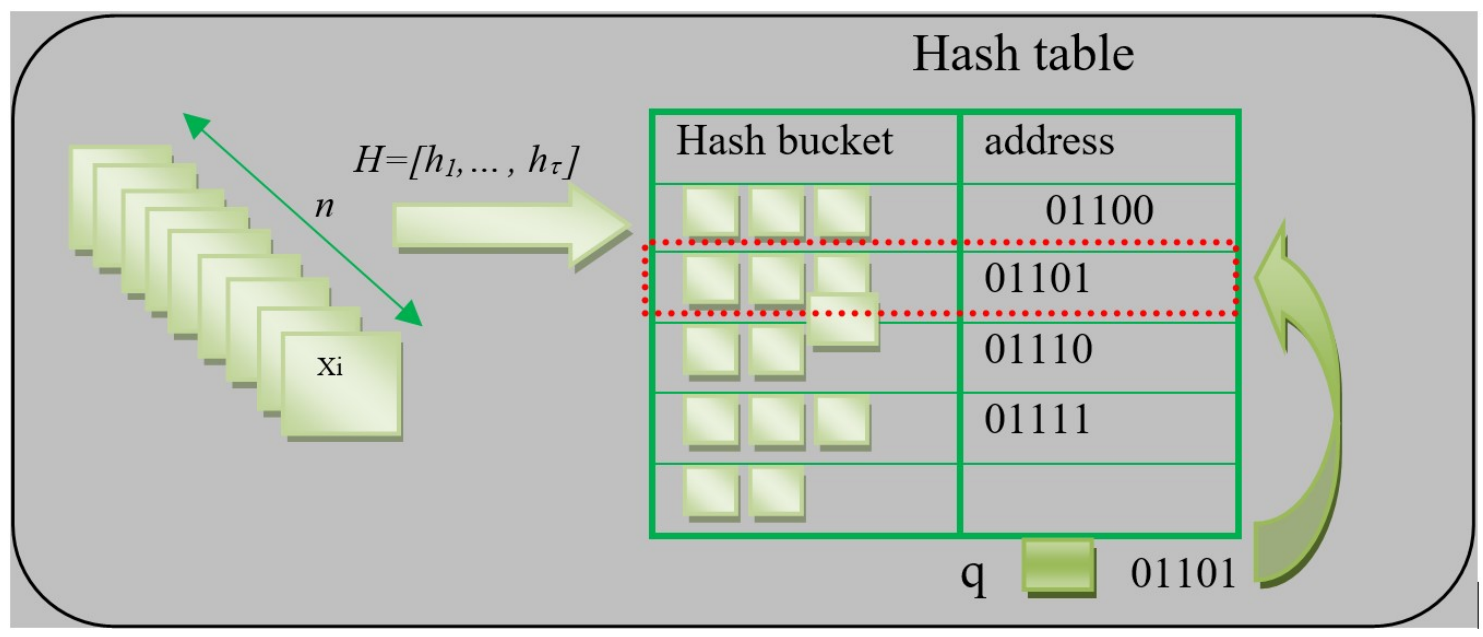

Figure 2. The Character Points Indexed by the Hash Table

The Figure 2 shows the hash table implemented after having the hash code. Note that a hash table indexes all the feature points we have in the database. Each square here represents a patch or characteristic or a sample. And for $\mathrm{n}$ samples, each of them will be mapped to a hash code [16].

\subsection{Facial Features Extraction}

The elementary component of how local feature is extracted and used to match images is also the one used to find similar images from a large database.

However, finding images from a database containing thousands of images and locations, where each image could involve hundreds of sites, hundreds of patches. This causes a great challenge as, on the side of "cloud server", we talk about millions of points or millions of "features" that must be stored and matched.

On the client side (the student), the extraction of "local feature" involves calculating the image processing. But to extract hundreds of "local features" and transmit them over the network, we will need a very quick response, this leads too many difficult problems when we design this search system such as: the power, memory and speed which are limited on mobile devices, limited bandwidth on the cellular network. So to handle all these difficult problems, there is a lot of research done in this field, 
not only on computer vision, image processing, feature extraction, but also on how this large dimensional feature is managed in real time.

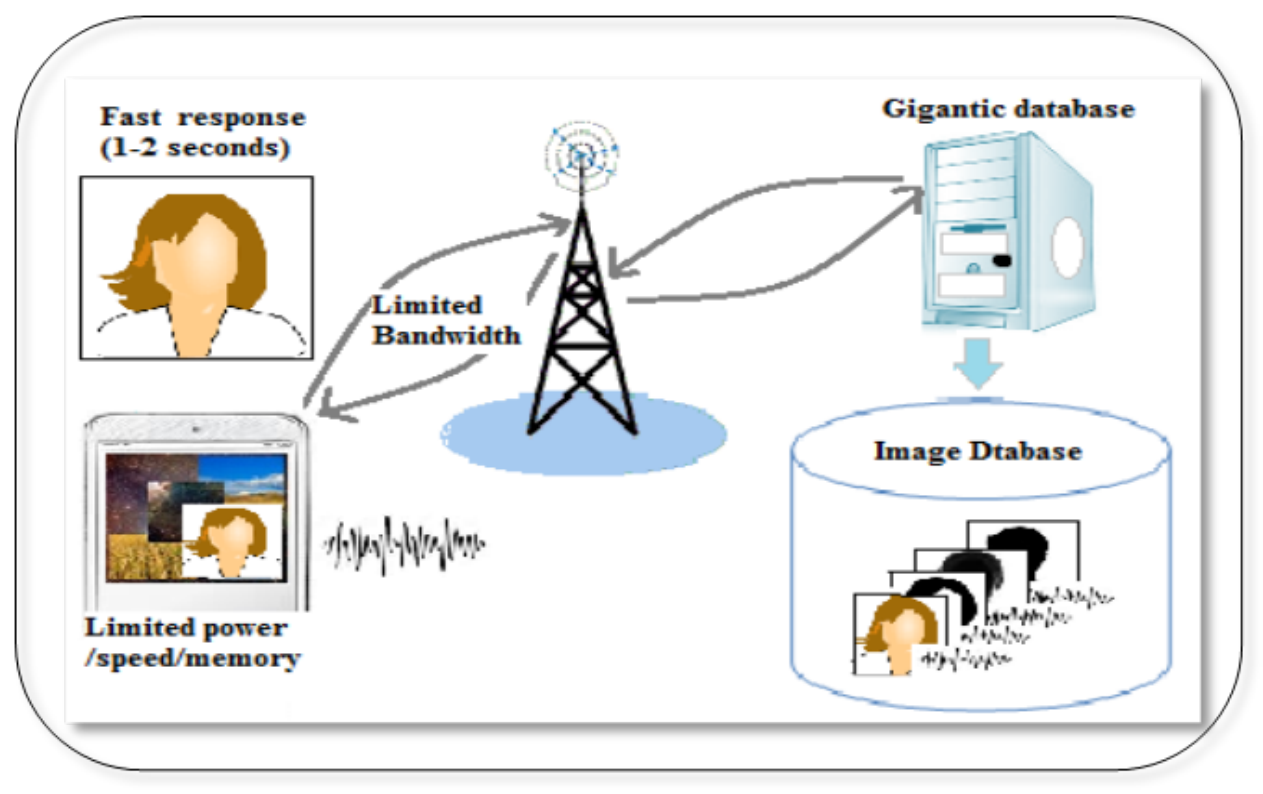

Figure 3. Facial Features Extraction in Our System

For each face detected, a partial descriptor extracted around certain facial features is used. First a generative model is developed to locate nine key points of the face, including the left and right corners of each eye, nostrils, tip of nose and left and right corners of the mouth. Then, we extract the SIFT descriptor from each key point and concatenate them to form our final face descriptor [17].

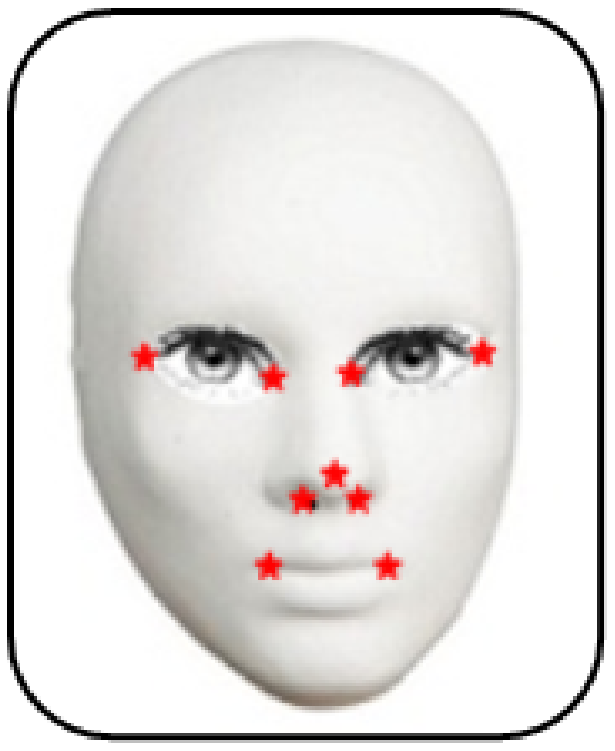

Figure 4. Examples of Facial Feature Points 


\section{The Predictive Model}

It is vastly recommended to provide learners with enough time to fill in the questionnaire. That is because it is essential to apprehend the learning style appropriate to each learner. The latter can even be presented with a personal profile that comprehends their strengths and weaknesses in a certain course or field.

For this reason, the following steps should be taken:

1. By relying on the J48 method, and through the usage of data collected from earlier students we form the central part of the model.

2. Have an estimation of the strengths and weaknesses of the new students through analysing the data collected from students who have already graduated.

3. Orienting learners towards the most appropriate profile that answers their motivation, and provide them with additional links to courses recommended by our system. These courses should be in different forms, such as videos, audios, texts, etc., depending on the student's learning style (Reflection, Reasoning, Sensory, and Progression).

4. Drawing a comparison between our findings and those predicted by the model, which would allow for further updating and improving of the latter

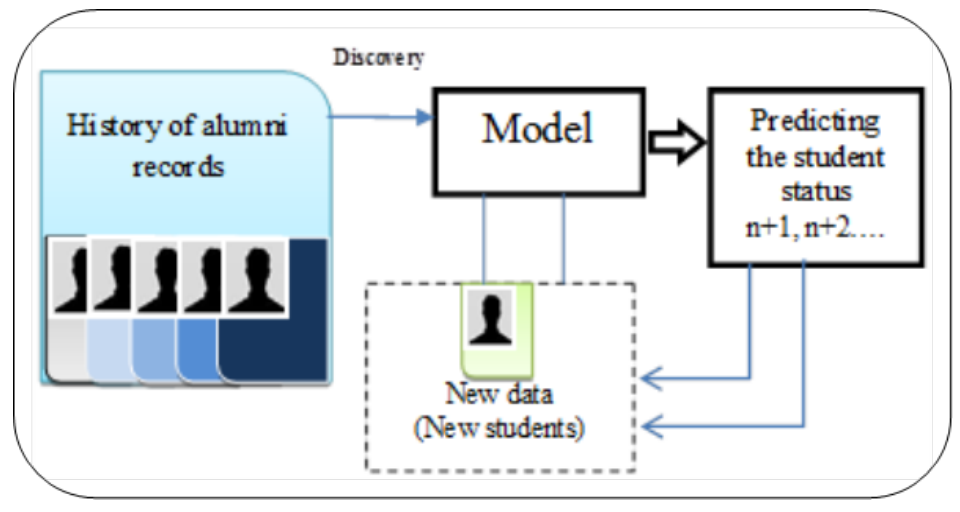

Figure 5. Predictive Model of Our System

\section{Performance and Evaluation}

In this article we searched the information of almost 3000 students of the Faculty of Charia described by 84 variables. These data are processed by several methods to achieve a model able to predict the strengths and weaknesses of new entrants. We present here the results of one of these methods of classification the $\mathrm{J} 48$ - which gave us good results.

In this study, we will calculate the test set using the execution components, for example, the order of accuracy and run time. In addition, we discover the precision measurement and the error rate.

The execution components for these arrangement calculations are shown in Table 1 and the precision measure per class for the classifier calculations is delineated in Table 2 .

Table 1. The Performance Factors for the Decision Tree J48

\begin{tabular}{c|c|c|c|c|c}
\hline \hline TP rate & Precision & F-Measure & $\begin{array}{c}\text { ROC } \\
\text { Curve }\end{array}$ & $\begin{array}{c}\text { Kappa } \\
\text { value }\end{array}$ & Execution time \\
\hline 0.676 & 0,664 & 0.677 & 0.675 & 0.2533 & 0.17 \\
\hline \hline
\end{tabular}


Table 2. Precision Measures for Decision Tree J48

\begin{tabular}{c|c}
\hline \hline Correctly classified & Incorrectly classified \\
\hline $67.6136 \%$ & $32.3864 \%$ \\
\hline
\end{tabular}

\section{Conclusion}

With explosive development of e-learner platform an efficient and accurate way to index and organize images according to the identities of the learner becomes heavily demanded.

Changes in the pose and illumination of the image are two main obstacles to the automatic recognition of the face. This is why we will need a new method for simultaneously handling the installation and lighting conditions.

This document opens the door to future research that may adopt other systems and methods to provide more realistic outcomes such as facial expression processing and analysis using sensors to determine the emotional states of e-learners. Or even detect the psychological state of an e-learner through his behaviour during the course. We can also improve our system by adding more devices that will help us to orient learners in their academic careers and even in the choice of the subject of their research projects.

\section{References}

[1] H. H. Alaoui, E. -K. Hachem, and C. Ziti, "Artificial Intelligence in e-Learning, chapter of Textbook: Shaping the Future of ICT: Trends," in Information Technology, Communication Engineering and Knowledge, published by CRC Press Taylor, 2017.

[2] H. H. Alaoui, E. -K. Hachem, and C. Ziti, "Artificial Intelligence," in e-Learning, Communication, Management and Information Technology - Sampaio de Alencar (Ed.), pp. 145-150. London: Taylor \& Francis Group, 2017.

[3] X. Ge, X. Huang, Y. Wang, M. Chen, Q. Li, T. Han and C. X. Wang, "Energy efficiency optimization for mimo-ofdm mobile multimedia communication systems with qos constraints," IEEE Trans on Vehicular Technology, vol. 63, no. 5, pp. 2127-2138, 2014.

[4] M. Turk and A. Pentland, "Eigenfaces for Recognition," J. Cognitive Neuroscience, vol. 3, no. 1, pp. 71-86, 1991.

[5] K. Etemad, and R. Chellappa, "Discriminant Analysis for Recognition Of Human Face Images," J. Optical Soc. Am, vol. 14, pp. 1724-1733, 1997.

[6] J. Yang, D. Zhang, A. F. Frangi and J. Yang, "Two-Dimensional PCA: A New Approach to Appearance-Based Face Representation and Recognition," IEEE Trans. Pattern Analysis and Machine Intelligence, vol. 26, no. 1, pp. 131-137, 2004.

[7] O. Arandjelovic, and A. Zisserman, "Who Are You? - Learning Person Specific Classifiers from Video," in Proceedings of the CVPR, pp. 1145-1152, 2009.

[8] J. Ji, S. Yan, J. Li, L. Gao, Q. Tian and B. Zhang, Batch-Orthogonal Locality-Sensitive Hashing for Angular Similarity," IEEE Trans on Pattern Analysis and Machine Intelligence, vol. 36, no. 10, pp. 1963-1974, 2014.

[9] X. Yuan, and S. Yan, Visual Classification with Multi-Task Joint Sparse Representation," in Proceedings of CVPR, pp. 3493-3500, 2010.

[10] B. Kavitha, S. Karthikeyan and B. Chitra, "Efficient Intrusion Detection with Reduced Dimension Using Data Mining Classification Methods and Their Performance Comparison," BAIP 2010, pp. 96-101, 2010.

[11] M. H. Danham, and S. Sridhar, Data Mining, Introductory and Advanced Topics. Person education, 2006.

[12] W. E. Spangler, J. H. May and L. G. Vargas, "Choosing Data-Mining Methods for Multiple Classification: Representational and Performance Measurement Implications for Decision Support," Journal of Management Information Systems, vol. 16, no. 1, pp. 37-62, 2015.

[13] P. Indyk and R. Motwani, "Approximate Nearest Neighbors: Towards Removing The Curse of Dimensionality," in ACM Symposium on Theory of Computing, 1998. 
[14] D. G. Lowe, "Object Recognition From Local Scale-Invariant Features," Computer Science Department University of British Columbia Vancouver, Canada, Proc. of the International Conference on Computer Vision, Corfu, Sept 1999.

[15] Q. Lv, W. Josephson, Z. Wang, M. Charikar, and K. Li, "Multi-Probe LSH: Efficient Indexing for High-Dimensional Similarity Search," in the proceedings of the 33rd International Conference on Very Large Data Bases, VLDB '07, VLDB Endowment, pp. 950-961, 2007.

[16] A. Gionis, P. Indyk, and R. Motwani, "Similarity Search in High Dimensions via Hashing," In the Proceedings of the 25th International Conference on Very Large Data Bases, VLDB'99, San Francisco, CA, USA. Morgan Kaufmann Publishers Inc, pp. 518-529, 1999.

[17] W. Zhao, R. Chellappa, J. Phillips, and A. Rosenfeld, "Face Recognition: A Literature Survey," ACM Computing Surveys, pp. 399-458, 2003. 